\title{
Theory of the structure of the self-trapped exciton in quartz
}

\author{
A J Fisher $\nmid$, W Hayes $\nmid$ and A M Stoneham $§$ \\ † Clarendon Laboratory, University of Oxford, Parks Road, Oxford OX1 3PU, UK \\ ¥ Theoretical Physics Division, Harwell Laboratory, Oxon OX11 ORA, UK \\ § Materials Physics and Metallurgy Division, Harwell Laboratory, Oxon OX11 ORA, \\ UK \\ || Department of Metallurgy and Science of Materials, University of Oxford, Parks \\ Road, Oxford OX1 3PH, UK
}

\section{Received 16 March 1990}

\begin{abstract}
Quartz is an insulator with an extremely wide band gap in the vacuum ultra-violet. However, under irradiation from high-energy electrons or $\mathrm{x}$-rays, samples of high purity emit a luminescence band in the blue, corresponding to a Stokes shift of approximately $7 \mathrm{eV}$. This large Stokes shift has been ascribed to the self-trapping of an exciton in an otherwise perfect lattice owing to the distortion it induces; we review the evidence for this assignment, and describe electronic-structure calculations which reveal the structure of the distorted configuration and also explain various experimentally determined properties of the centre. The self-trapping process we postulate is a novel one as it is driven primarily by the electron component of the exciton.
\end{abstract}

\section{Introduction}

Quartz is one of the most familiar of crystals. It is the most stable allotrope at room temperature and pressure of silicon dioxide, which is a compound of great mineralogical and technological importance, and quartz itself has been much studied in recent years because of its use in the production of oscillators for timing devices. Yet a number of puzzles still remain in its behaviour, and this paper describes theoretical work undertaken in an attempt to resolve one such outstanding problem.

Quartz is a material with, loosely speaking, mixed ionic and covalent character. Its local structure is characteristic of covalent materials, each silicon atom being surrounded by four oxygen atoms in a configuration which is slightly distorted from a perfect tetrahedron (figure 1). This suggests that the local bonding forces are predominantly covalent, dominated by the $\mathrm{sp}^{3}$ hybridisation of the silicon atoms. However, the electronegativities of silicon and oxygen, on any of the many scales of measurement available (see Catlow and Stoneham 1983 for a discussion), are very different. This difference gives the lattice a substantial ionic character, which is reflected experimentally in a difference between the static and optical dielectric constants, which are around 4.5 and 2.4 respectively (Hellwege and Hellwege 1979). Electronic structure calculations show substantial charge transfer from silicon to oxygen (see for example Chelikowsky and Schlüter 1977).

The band-gap in quartz is large and because it is relatively inaccessible (in the vacuum ultra-violet) there is some experimental uncertainty in its value. However, 


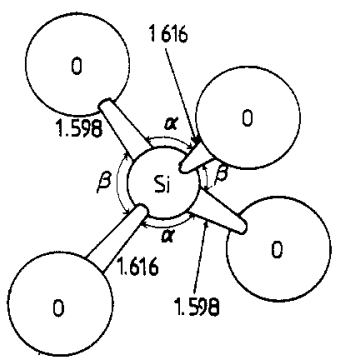

Figure 1. A single $\mathrm{SiO}_{4}$ tetrahedron as found in the $\alpha$-quartz structure. The bond lengths are indicated in $\AA$. The angles $\alpha$ and $\beta$ are $108.4^{\circ}$ and $110.4^{\circ}$, respectively, while the angles (not shown) between the two long bonds and between the two short bonds are $110.9^{\circ}$ and $108.4^{\circ}$, respectively. The tetrahedra are packed so that each oxygen atom is bonded to two silicons, to one by a short bond and to the other by a long bond.

the gap is believed to be indirect and approximately $11 \mathrm{eV}$ wide (Klein and Chun 1972), although there is substantial free-exciton absorption at lower energies. The gap is thought to be primarily ionic in nature, the top of the valence band comprising non-bonding oxygen $\mathrm{p}$ states while the conduction band consists mainly of silicon $\mathrm{s}$ states. However there seems to be a finite admixture of silicon-oxygen anti-bonding character into the conduction band (Chelikowsky and Schlüter 1977), and this has important consequences for the following discussion.

When high-purity quartz crystals are subjected to above-band-gap irradiation, and electron-hole pairs are produced, a strong luminescence band is seen in the blue at energies just below $3 \mathrm{eV}$. This represents an exceptionally large Stokes shift relative to the band gap; we shall be concerned here with the relaxation responsible for the Stokes shift, and with the nature of the excited state produced by the relaxation. First (in section 2) we shall describe in more detail a wide variety of experimental results which indicate that the excited state may be considered to be a triplet exciton which has selftrapped because of the lattice distortion it produces. Then we shall discuss electronic structure calculations, first (briefly) of the semi-empirical type (subsection 3.1) and then (more extensively) at the $a b$ initio Hartree-Fock level (subsection 3.2). Finally (section 4) we shall compare predictions of the $a b$ initio calculations with experimental results. A short account of this work has been given elsewhere (Fisher et al 1990).

\section{Review of experimental results}

The blue luminescence in quartz has been known experimentally for a number of years (Sigel 1973). It was decomposed into three overlapping peaks with different thermal quenching characteristics by Alonso et al (1983) and by Tanimura and Halliburton (1986); their energies and quenching temperatures are shown in table 1. Band III (table 1) can be removed by $x$-irradiation at room temperature and its thermal quenching is correlated with the onset of thermally-activated hopping of alkali metal ions (Halliburton et al 1981); it has been assigned to recombination at an aluminium-related hole centre whose charge is compensated by an alkali metal ion. Itoh et al (1988) have observed a sub-linear dependence of the intensity of the band I luminescence (table 1) on incident radiation flux and, on the assumption that this represents the saturation of 
the recombination rate at an impurity, have concluded that this band is also extrinsic in origin. The band II luminescence, centred around $2.78 \mathrm{eV}$, is seen in all samples and has a linear dependence on the incident flux even when the latter becomes large (Itoh et al 1988); there are therefore strong reasons for supposing this contribution to be an intrinsic phenomenon of the quartz crystal, as suggested by Trukhin and Plaudis (1979).

Table 1. Characteristics of the overlapping bands contributing to the blue luminescence.

\begin{tabular}{lcl}
\hline Band & $\begin{array}{l}\text { Quenching } \\
\text { temperature }(\mathrm{K})\end{array}$ & $\begin{array}{l}\text { Energy } \\
\text { of peak }(\mathrm{eV})\end{array}$ \\
\hline I & 130 & 2.48 \\
II & $>160$ & 2.78 \\
III & 220 & 3.26 \\
\hline
\end{tabular}

The blue luminescence is linked to a transient deformation of the crystal. This was first demonstrated by Tanimura et al (1983), who were able to detect a transient volume change by the change in the optical rotation (Tanimura et al 1984). They found that a transient volume increase with a magnitude of approximately one $\mathrm{SiO}_{2}$ molecular volume per emitted photon occurred when the crystal was subjected to above-band-gap irradiation; the exponential decay of this expansion, with a time constant of approximately $1 \mathrm{~ms}$, was correlated with the decay of the blue luminescence. Similar effects were observed in amorphous silica and in aluminium oxide (Itoh et al 1986); in the latter case the Stokes shift was substantially smaller than in quartz as the luminescence band occurred in the ultra-violet at around $7.5 \mathrm{eV}$, compared with a band gap of $8.3 \mathrm{eV}$. A further indication that production of free carriers in quartz can have a strong effect on the lattice is provided by the work of Hobbs and Pasucci (1980), who carried out transmission electron microscopy with the electron beam producing irradiation in situ. They observed the nucleation of strain centres within the crystal and an eventual crystalline-to-amorphous transition of the whole sample and found that both processes scaled with the ionisation cross-section, not the displacement cross-section, of the incident electrons; they therefore concluded that radiolytic processes were responsible. More recently, Howitt et al (1990) have shown that bombardment by ions, which produce primarily displacement damage, and by electrons, which produce primarily ionisation damage, both induce such a transformation at a rate which is linearly proportional to the dosage. They find no distinction between the type of damage produced by the ions and by the electrons and suggest that electron bombardment produces oxygen vacancies as the end point of a radiolytic process. While none of these observations prove that exciton self-trapping is involved, they all suggest that large lattice distortions are involved in the bulk recombination processes.

Direct probes of the nature of the excited state responsible for the volume change are provided by spin resonance techniques, particularly by optically detected magnetic resonance (ODMR). In this method resonances that alter the relative occupancies of states within an excited manifold are detected by changes in the intensity of allowed optical transitions to the ground state (Davies 1976). Experiments of this type show that the state whose decay produces the blue luminescence is a triplet with exceptionally large fine structure (Hayes et al 1984). The fine structure may be fitted to a 
Table 2. ODMR principal axes for the fine structure of the intrinsic blue luminescence (from Hayes and Jenkin 1988).

\begin{tabular}{llr}
\hline & $\begin{array}{l}\theta \\
\text { Axis }\end{array}$ & $\begin{array}{l}\phi \\
\text { (deg) }\end{array}$ \\
\hline$\rho$ & 85 & 85 \\
$\eta$ & 45 & 350 \\
$\xi$ & 45 & 180 \\
\hline
\end{tabular}

standard spin Hamiltonian of the form

$$
\hat{H}_{\mathrm{fs}}=D\left[\left(S_{\rho}^{2}-\frac{1}{3} S(S+1)\right]+E\left(S_{\xi}^{2}-S_{\eta}^{2}\right)\right.
$$

where $S$ is the total spin of the system and $\left(S_{p}, S_{\eta}, S_{\xi}\right)$ are its components along the principal axes $(\rho, \eta, \xi)$ of the fine structure tensor (see table 2). The parameters $D$ and $E$ are then found to take the values $\pm 22.6 \mathrm{GHz}$ and $\mp 1.6 \mathrm{GHz}$ respectively (only the relative signs are known and the overall sign has not been determined). The presence of a triplet state implies that at least two carriers are involved, and further suggests that these observations may be interpreted in terms of the localisation of excitons.

There are also optical absorption lines associated with the excited triplet state. Tanimura et al (1983) found that a transient absorption line at $5.4 \mathrm{eV}$ is associated with both the blue luminescence and the transient volume change, while more recently correlation with a second absorption line, at $4.2 \mathrm{eV}$, has been demonstrated (Itoh et al 1988). Bleaching of the crystal with laser light of energy between $3.0 \mathrm{eV}$ and $5.0 \mathrm{eV}$ after irradiation destroys the blue luminescence.

Finally we mention two further properties of the luminescence. The first is its polarisation; Tanimura and Halliburton (1986) found that the $2.78 \mathrm{eV}$ component has polarisation +0.48 parallel to the crystal $c$ axis when viewed in the $a b$ plane, while Itoh et al (1988) studied only those components of the luminescence decaying with the relaxation time of the transient volume change and found a polarisation of +0.65 parallel to the $c$ axis. The second property is the excitation spectrum for the production of the blue luminescence; this has been measured by Trukhin and Plaudis (1979) and by Itoh et al (1989a) and shows a strong peak at around $9 \mathrm{eV}$, near the onset of free-exciton absorption.

\section{Electronic structure calculations}

\subsection{Semi-empirical calculations and the peroxy model}

We begin our theoretical discussion of the structure of the excited triplet state with a brief description of the results we have obtained at Harwell using the MOSES semiempirical Hartree-Fock package (Harker and Lyon 1979). These initial calculations were motivated by the experimental evidence suggesting that the excited state has the structure of a close oxygen-oxygen pair or peroxy linkage associated with a nearby oxygen vacancy; this evidence includes the similarity of the large uniaxial fine structure to the fine structure of molecular oxygen (Tinkham and Strandberg 1954) and the resemblance of the $5.4 \mathrm{eV}$ absorption line to the $5.8 \mathrm{eV}$ absorption in the oxygen vacancy or E-centre which has been known in quartz for some years (Tanimura et al 1983, Weeks 1956, Rudra and Fowler 1987). We have reported elsewhere (Fisher et al 
1989, Fisher 1989) some of our calculations designed to test this model; in particular, we found that the fine structure to be expected from an $\left(\mathrm{O}_{2}\right)^{2-}$ molecular ion is similar to that observed in quartz if the interatomic axis is identified with the fine-structure principal axis. The polarisation of the luminescence would also be similar to that observed experimentally.

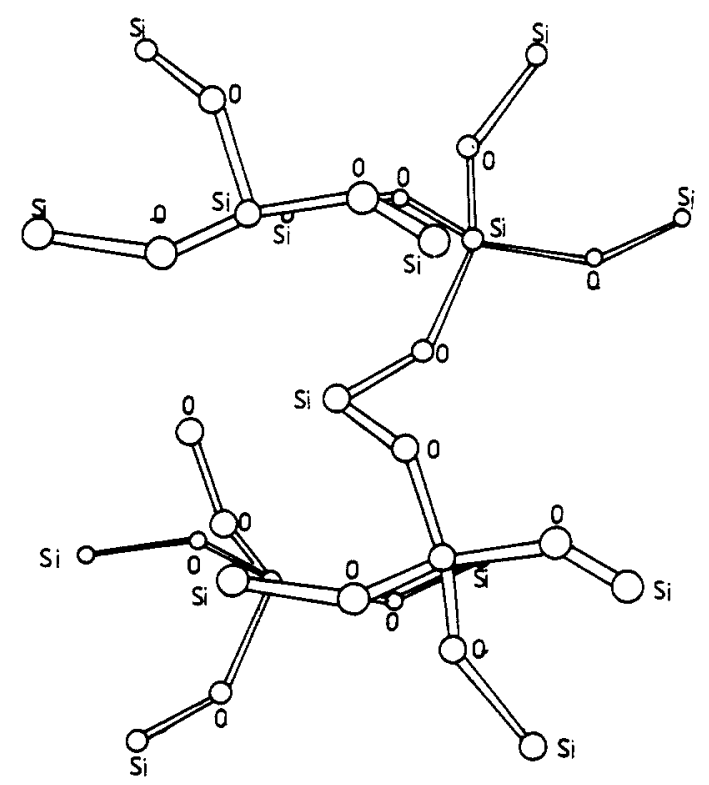

Figure 2. Configuration of minimum energy obtained by allowing motion of two oxygen atoms in the INDO approximation. Note the formation of a peroxy linkage between the two oxygen atoms.

Our semi-empirical electronic structure calculations seemed at first sight to bear out this model, since we find that the configuration, of minimum energy in both the sc CNDO and INDO approximations (Pople and Beveridge 1970) contains a close oxygen pair (figure 2). However, these results show the highly unphysical feature of the singlet ground state in this atomic configuration, having an energy some $20 \mathrm{eV}$ lower than the perfect crystal. This indicates that the semi-empirical methods, with the parametrisation we have employed, artificially favour oxygen-oxygen bonding; similar effects have been observed in work on intermolecular interactions in water, where the stable configuration for a dimer was found to involve the close approach of two oxygen atoms rather than the expected hydrogen bonding (Thiel 1978). In this case, an $a b$ initio Hartree-Fock calculation revealed that the energy minimum involving the oxygen pair was purely an artefact of the semi-empirical approximation.

The uncertainty involving the semi-empirical calculations is reinforced by the very different conclusions to which other workers have been led using similar, but differently parametrised, methods. Calculations using the INDO method with a parameter set specifically designed to reproduce the bulk properties of $\mathrm{SiO}_{2}$ have been carried out on the ideal cubic $\beta$-cristobalite structure, in which all the silicon-oxygen bonds are equivalent. Shluger (1988) reported a cluster calculation, while in a subsequent paper (Shluger and Stefanovich 1989) the higher symmetry of the cristobalite lattice enabled the authors to employ a supercell geometry. It was found that for the triplet spin configuration a metastable state of the lattice existed that could be produced, to a good 
approximation, just by rotating an oxygen atom about one of the other silicon-oxygen bonds of a neighbouring silicon atom in such a way that one of the oxygen-oxygen distances was reduced from $2.5 \AA$ to $2.2 \AA$. The oxygen pairing is much less pronounced than predicted by our own semi-empirical calculations and the electron states are associated primarily with individual atoms, rather than with a peroxy linkage.

\subsection{Full Hartree-Fock calculations}

In view of the differing answers obtained using two semi-empirical parametrisations, we now describe $a b$ initio calculations at the Hartree-Fock level, carried out in clusters of atoms designed to simulate the atomic environment in $\alpha$-quartz. We have chosen a Hartree-Fock, rather than a density functional, method for two reasons. First, we need to perform calculations in the triplet state; much less experience is available with spin-density functional methods than with the charge-density techniques that suffice for singlet states. Secondly, extraction of two-electron matrix elements such as the fine-structure parameters from a density functional calculation, which is designed to reproduce the ground state charge or spin density without regard for electron-electron correlations, involves non-trivial issues of principle.

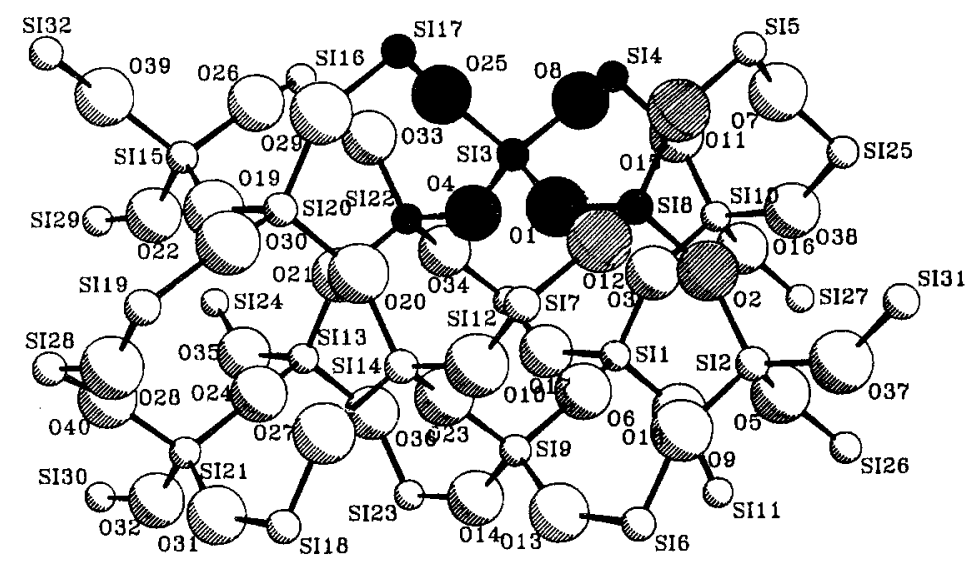

Figure 3. The clusters used in the Hartree-Fock calculations, shown in the quartz lattice viewed along the $y$-axis. The atoms forming the silicon-centred cluster are shaded black, while the atoms in the oxygen-centred but not the silicon-centred cluster are hatched.

Two clusters were used in this work; both contained nine atoms but one was centred on a silicon site and one on an oxygen site. The clusters are shown in position in the quartz lattice in figure 3. The small size treated is due to the large memory requirement for the storage of the two-electron matrix elements of the Coulomb interaction. The calculations were performed using the CADPAC program (Amos and Rice 1987), which is able to perform open and closed shell Hartree-Fock calculations and also includes, among other facilities, the analytic (i.e. not involving numerical differencing) evaluation of the first and second derivatives of the total energy with respect to the atomic displacements, the use of these derivatives in an efficient geometry optimisation algorithm, and the ability to surround a cluster which is treated quantum mechanically by an array of ions in order to simulate the electric field of a crystal. For most of the calculations described here, the simplest of the standard 
basis sets available to CADPAC was used, namely the so-called STO-3G basis which is roughly equivalent to a minimal basis of Slater-type orbitals, with the radial part of each STO represented by a contraction of three Gaussians. The array of ions surrounding the central cluster was obtained by dividing the quartz lattice into neutral $\mathrm{SiO}_{4}$ tetrahedra in which the silicon atom carries charge +4 and the oxygen atoms charge -1 and including all those tetrahedra for which the central silicon lies within 15 Bohr radii $(6.94 \AA)$ of the central atom of the quantum mechanical cluster. Throughout the interior of the ionic array two tetrahedra overlap at each oxygen site thereby giving the expected oxygen charge of -2 , but at the outer edges of the array the oxygen charge is -1 , ensuring overall charge neutrality. No relaxation of the outer ionic array or of the outermost atoms in the quantum mechanical cluster was permitted during minimisation of the energy.

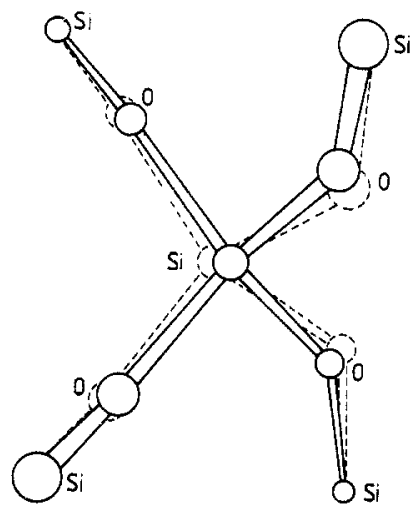

Figure 4. The silicon-centred cluster after relaxation in the singlet spin state subject to the constraint that the crystalline $C_{2}$ rotation axis should be retained. The perfect-crystal positions of the atoms are shown by broken lines.

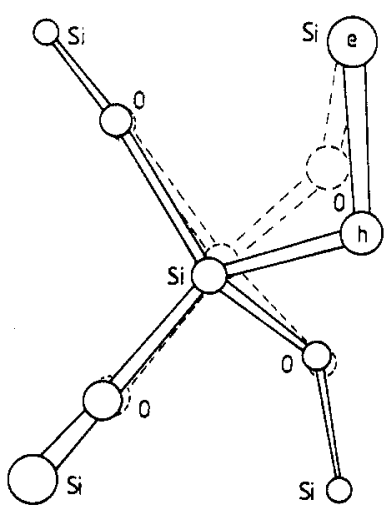

Figure 5. The silicon-centred cluster after relaxation in the triplet state with no symmetry restrictions, corresponding to the self-trapping of an exciton. The atoms on which the electron and hole components are primarily localised are indicated by the letters e and $h$ respectively. The optimum positons of the atoms in the singlet state are shown by broken lines.

First we describe our calculations on the silicon-centred cluster, consisting of a central silicon atom, its four oxygen neighbours (all of which were allowed to relax), and the four additional silicon neighbours of these oxygen atoms. This cluster is shown with the atoms in their perfect-crystal positions in figure 4 (broken lines). When the atoms were permitted to relax in the singlet spin state subject to the restriction that the $C_{2}$ rotation symmetry about the $x$-axis be retained, small displacements occurred to give the configuration shown in figure 4 (full lines), with a total energy gain of $0.87 \mathrm{eV}$; this shows that the minimum-energy configuration of the cluster is close to the perfect crystal structure. Next the cluster was excited into a triplet state in order to simulate the presence of a triplet exciton, and unconstrained relaxation of the five central oxygen atoms was permitted after an initial symmetry-breaking displacement of the type suggested by Shluger (1988). A much larger displacement of the atoms from the perfect crystal positions was now produced, concentrated at a single oxygen site; the final configuration, produced when all components of the force on each relaxing atom had dropped below $0.001 \mathrm{Hartree}^{\mathrm{Bohr}} \mathrm{H}^{-1}\left(0.051 \mathrm{eV} \AA^{-1}\right)$, is 
shown in figure 5 . The energy gained relative to the triplet state in the perfect crystal geometry by making this relaxation was $2.6 \mathrm{eV}$. In this geometry the hole component of the triplet exciton is primarily concentrated on the displaced oxygen atom $(92 \%$ of the charge density) while the electron component is primarily concentrated on the silicon atom to which this oxygen was short-bonded in the perfect crystal, and from which it has moved away during the relaxation process. Some of the electron spin density (about $30 \%$ of the charge density) is also found on the displaced oxygen atom. The singlet-triplet excitation energy is now reduced to $1.9 \mathrm{eV}$.

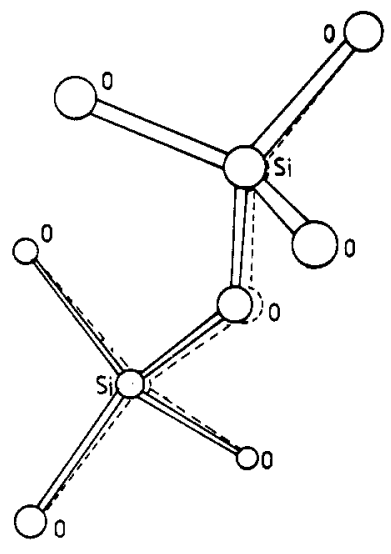

Figure 6. The oxygen-centred cluster after relaxation in the singlet spin state. The perfectcrystal positions of the atoms are shown by broken lines.

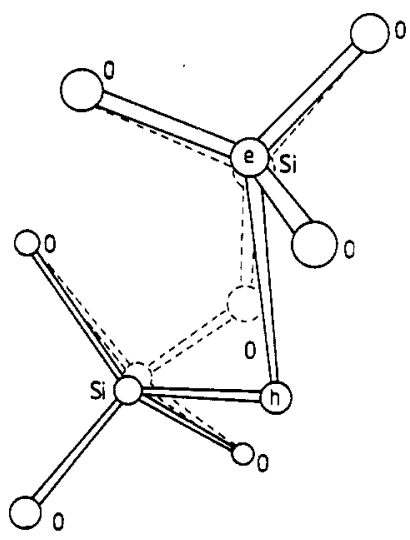

Figure 7. The oxygen-centred cluster after relaxation in the triplet state with no symmetry restrictions, corresponding to the self-trapping of an exciton. The atorns on which the electron and hole components are primarily localised are indicated by the letters $e$ and $h$ respectively. The perfect-crystal positions of the atoms are shown by broken lines.

Next we describe complementary calculations performed on an oxygen-centred cluster consisting of a central oxygen atom, its two silicon nearest neighbours, and six further oxygen atoms (three bonded to each of the silicon neighbours). The atoms are shown in their perfect-crystal positions in figure 6 (broken lines). When the atoms were allowed to relax in the singlet state (this time without any symmetry restrictions since the cluster is not left invariant under any of the operations of the crystal point group) the small displacements shown in figure 6 occurred, with an energy gain of $0.25 \mathrm{eV}$. In the perfect-crystal geometry the excitation energy from the singlet ground state to the first triplet state was $13.3 \mathrm{eV}$; on moving the central oxygen atom of the cluster and one of its silicon neighbours to the positions found to be optimal for the triplet state in the silicon-centred cluster calculations, the energy of the triplet state was reduced by $3.8 \mathrm{eV}$. When the three central atoms (one oxygen and two silicon) of the present oxygen-centred cluster were allowed to relax further in the triplet state, there was a further gain in energy of $1.8 \mathrm{eV}$ and the equilibrium configuration shown in figure 7 was produced. In this configuration, the singlet-triplet excitation energy is reduced to $1.5 \mathrm{eV}$. The principal difference between this geometry and that produced by optimisation of the silicon-centred cluster is that the oxygen atom has now moved further away from its perfect-crystal position; we shall see below that this difference has rather little impact on the predictions made from the two different geometries for 
experimentally observable quantities. Once again the hole component of the exciton is localised ( $92 \%$ of the charge density) on the oxygen atom which makes the largest displacement from its perfect-crystal position, while the electron component is located primarily on the silicon atom to which this oxygen atom was short-bonded in the perfect crystal, but also partly on the oxygen atom itself (15\% of the charge density). The coordinates of the central oxygen atom and its two silicon neighbours before and after the relaxations in the two different cluster shapes are shown in table 3.

Table 3. Positions of atoms before and after relaxation in the triplet state. All lengths in atomic units.

\begin{tabular}{lllll}
\hline Atom & Coordinate & Perfect crystal & $\begin{array}{l}\text { Si-centred } \\
\text { relaxation }\end{array}$ & $\begin{array}{c}\text { O-centred } \\
\text { relaxation }\end{array}$ \\
\hline $\mathrm{O}$ & $x$ & 2.590 & 2.263 & 2.059 \\
& $y$ & 2.187 & 1.782 & 1.954 \\
& $z$ & 1.226 & 0.512 & 0.295 \\
$\mathrm{Si}$ & $x$ & 4.317 & 4.568 & 4.446 \\
& $y$ & 0.000 & 0.249 & 0.180 \\
& $z$ & 0.000 & 0.209 & 0.184 \\
$\mathrm{Si}$ & $x$ & 2.483 & Not relaxed & 2.500 \\
& $y$ & 4.301 & Not relaxed & 4.457 \\
& $z$ & 3.404 & Not relaxed & 3.574 \\
\hline
\end{tabular}

That the essential features of the geometry remain unaltered in two calculations with clusters of completely different shapes indicates that cluster shape does not have a strong influence on our results. We have performed additional calculations to check for dependence on the basis set, and on the size and the charge of the point-ion lattice used to mimic the electric field of the bulk quartz crystal. In each case the oxygencentred cluster was used and the gradient of the total energy was evaluated using the revised parameters but in the minimum-energy geometry shown in figure 7 . When the basis set was altered from STO-3G to STO-4G (so that each Slater-type orbital was represented by a contraction of four, rather than three, Gaussians) the singlet-triplet excitation energy became $1.4 \mathrm{eV}$ and the largest component of the energy gradient in the triplet state was $0.002 \mathrm{Hartree} \mathrm{Bohr}^{-1}$. When all of the charges in the pointion array surrounding the quantum cluster were halved, the singlet-triplet excitation energy became $2.1 \mathrm{eV}$ and the largest component of the energy gradient in the triplet state was 0.024 Hartree Bohr $^{-1}$. When the point-ion array, with the original charges, was enlarged to include all $\mathrm{SiO}_{4}$ tetrahedra with $\mathrm{Si}$ atoms lying within $20.0 \mathrm{Bohr}$ of the central oxygen atom, the singlet-triplet excitation energy became $1.9 \mathrm{eV}$ and the largest component of the energy gradient in the triplet state was $0.024 \mathrm{Hartree} \mathrm{Bohr}^{-1}$. In all cases the forces which arise in the original minimum-energy geometry due to the change in the parameters are small, and give no grounds for believing that a full optimisation of the geometry using different parameters would lead to significantly different results.

\section{Comparison with the experimental results}

We now compare the predictions of the optimised triplet geometry with experimentally-observed characteristics of the self-trapped exciton in quartz. 


\subsection{The luminescence energy}

The calculated energy differences between the triplet and singlet states in the minimum-energy geometries just described are $1.9 \mathrm{eV}$ and $1.5 \mathrm{eV}$ for the Si-centred and $\mathrm{O}$-centred clusters respectively. These are somewhat smaller than the observed luminescence energy of $2.8 \mathrm{eV}$ but, given the established difficulty in estimating the energies of excited states, this error seems acceptable. The Stokes shifts predicted by our calculations are $74 \%$ of the theoretical band gap for the Si-centred cluster and $89 \%$ for the O-centred cluster, while the experimental value is $69 \%$.

\subsection{The radiative lifetime}

The observed lifetime of $1 \mathrm{~ms}$ is typical for a spin-forbidden transition in the visible region of the spectrum and so will be correctly predicted by almost any model, including ours.

\subsection{The fine structure}

There are two principal sources for fine structure in non-magnetic systems which are without rotational symmetry, so that the orbital angular momentum is quenched (Stoneham 1975): the first is the spin-orbit coupling taken to second order in perturbation theory and the second is the magnetic dipole-dipole interaction between the spins of the carriers. Noting that the spin-orbit coupling is a rapidly increasing function of the atomic number, but that very similar fine-structure parameters have been observed for an exciton in quartz trapped at a germanium impurity (Hayes and Jenkin 1988) whose atomic number is much larger, we ascribe the fine structure of the self-trapped exciton to the dipole-dipole interaction. The fine-structure tensor then has the form (in SI units):

$$
D_{i j}=\frac{1}{2} \frac{g^{2} \mu_{\mathrm{B}}^{2} \mu_{0}}{4 \pi}\left\langle\psi(1,2)\left|\frac{\delta_{i j} r^{2}-3 r_{i} r_{j}}{r^{5}}\right| \psi(1,2)\right\rangle
$$

where $\psi(1,2)$ is the spatial part of the two-electron wavefunction, $\mu_{\mathrm{B}}$ is the Bohr magneton and $\boldsymbol{r}$ is the displacement vector separating the two carriers. Contributions to the tensor decrease as the inverse cube of the distance between the carriers, so we would expect the expectation value to be dominated by that part of the wave-function in which the two carriers are both found on the displaced oxygen atom, with a smaller correction term arising from the interaction between the hole on the oxygen and the electron on the neighbouring silicon. These atoms are sufficiently distant that the detailed structure of the orbitals on the two atoms are unimportant in computing the correction and the carriers can be taken to be localised at the respective nuclei without any loss of accuracy (Stoneham 1975). The calculation of the contribution from two electrons on the same atom is greatly simplified by the rotational symmetry of the free atom; the Wigner-Eckart theorem enables all the contributions to be written in terms of the reduced matrix elements of the tensor operator representing the spin-spin interaction between two-electron atomic states with either $\mathrm{s}$ or $\mathrm{p}$ symmetry. These reduced matrix elements can either be evaluated directly from the forms for the basis functions used by the CADPAC program, or may be estimated from the deviations from the Landé interval rule in atomic spectra (Horie 1953, Sobel'man 1972); in either case, 
Table 4. Fine-structure parameters from calculation and experiment.

\begin{tabular}{|c|c|c|c|c|c|}
\hline & \multirow{2}{*}{$\begin{array}{l}D \\
(\mathrm{GHz})\end{array}$} & \multirow{2}{*}{$\begin{array}{l}E \\
(\mathrm{GHz})\end{array}$} & \multicolumn{3}{|c|}{ Principal axes } \\
\hline & & & & $\theta(\mathrm{deg})$ & $\phi(\mathrm{deg})$ \\
\hline Observed & \pm 22.6 & $\mp 1.6$ & $\begin{array}{l}\rho \\
\eta \\
\xi\end{array}$ & $\begin{array}{l}85 \\
45 \\
45\end{array}$ & $\begin{array}{r}-35 \\
230 \\
60\end{array}$ \\
\hline $\begin{array}{l}\text { Si-centred } \\
\text { cluster }\end{array}$ & -40.8 & 9.8 & $\begin{array}{l}\rho \\
\eta \\
\xi\end{array}$ & $\begin{array}{l}81 \\
34 \\
57\end{array}$ & $\begin{array}{r}-29 \\
254 \\
55\end{array}$ \\
\hline $\begin{array}{l}\text { O-centred } \\
\text { cluster }\end{array}$ & -18.1 & 3.0 & $\begin{array}{l}\rho \\
\eta \\
\xi\end{array}$ & $\begin{array}{r}85 \\
174 \\
86\end{array}$ & $\begin{array}{r}-38 \\
-1 \\
52\end{array}$ \\
\hline
\end{tabular}

the magnitudes are found to be between $300 \mathrm{GHz}$ and $500 \mathrm{GHz}$. The resulting finestructure parameters from the calculation and from experiment are shown in table 4 .

The comparison is satisfactory for the major principal axis (the $\rho$ axis), which dominates the observed splitting. The minor axes are also given quite well by the Si-centred calculation. We stress that in the model we propose the major principal axis of the fine structure is not associated with any inter-atomic direction, but instead arises from the anisotropic occupancy of the $\mathrm{p}$ orbitals on the displaced oxygen atom.

\subsection{The polarisation of the luminescence}

When observed along a twofold axis of the crystal, the intrinsic component of the blue luminescence has been reported to possess a polarisation parallel to the $c$-axis of +0.48 (Tanimura and Halliburton 1986) and +0.65 (Itoh et al 1988). We estimate the polarisation to be expected in the present model most simply if we note that the transition is mostly a charge transfer of the electron component of the exciton from the silicon atom to the oxygen and the dipole vector is therefore proportional to the inter-atomic displacement vector. On this basis, we calculate the polarisation to be +0.44 from the Si-centred cluster and +0.65 from the O-centred cluster. We point out that a more exact calculation is not feasible since it would involve evaluating the dipole matrix element between the singlet ground state and the singlet admixture which is introduced into the triplet state by the spin-orbit coupling and through which the spin-forbidden transition is mediated.

\subsection{The transient volume change}

Strictly, for a charged defect involving a long-range polarisation field and associated distortion in the lattice, the derivative of the free energy of formation with respect to pressure is required to make an accurate calculation of the volume of formation (Gillan 1981, Lidiard 1981, Stoneham 1983). However, for a neutral defect a simpler expression derived using the Betti reciprocity theorem for a harmonic crystal may be used (Temkin 1970). This expresses the volume change caused by the defect in the following form:

$$
\Delta V=\sum_{i, j, k} s_{i j k l} G_{i j}
$$


where $s_{i j k l}$ is a component of the elastic compliance of the crystal and $G_{i j}$ is the force dipole or virial caused by the presence of the defect. We have taken published values for the six independent compliances (Hellwege and Hellwege 1979) and estimated the virial by summing the difference between the forces on the nine atoms in the triplet state and the singlet state. In table 5 we give the results for both the Si-centred and the $\mathrm{O}$-centred clusters in the perfect-crystal geometry and the geometry obtained by relaxing the triplet state as described above; the consistency of the results for the different shapes of cluster and different geometries are an indication that this simple theory is approximately valid and the agreement with the experimentally-observed number (Tanimura et al 1983, 1984), in which the uncertainty is rather large, is good.

Table 5. Transient volume changes (in units of the $\mathrm{SiO}_{2}$ molecular volume) from theory (this work) and experiment (Tanimura et al 1983, 1984).

\begin{tabular}{lr}
\hline 1. Si-centred cluster, perfect crystal forces & +1.55 \\
2. Si-centred cluster, relaxed exciton forces & +0.99 \\
3. O-centred cluster, perfect crystal forces & +2.08 \\
4. O-centred cluster, relaxed exciton forces & +1.18 \\
Experimental: & $\simeq+1.0$
\end{tabular}

\subsection{The excitation spectrum}

It is not usually possible to converge an SCF calculation to an excited state if it has the same symmetry as a lower-energy state. We therefore have to rely on one-electron energy differences to estimate the excitation energies of the relaxed triplet exciton. For the Si-centred cluster we find a one-electron excitation energy for the electron and hole components of $4.9 \mathrm{eV}$ and $6.1 \mathrm{eV}$ respectively while for the O-centred cluster these numbers are $7.3 \mathrm{eV}$ and $5.7 \mathrm{eV}$. These results are in fair agreement with the observed peaks in the excitation spectrum at $4.2 \mathrm{eV}$ and $5.2 \mathrm{eV}$ (Tanimura et al 1983, Itoh et al 1988), although they do not allow us to distinguish which peak should be assigned to electron excitations and which to the hole.

\section{Conclusions}

We have performed $a b$ initio cluster calculations at the Hartree-Fock level which show that self-trapping of a triplet exciton, involving the displacement of a single oxygen atom of the type postulated by Shluger (1988) in the $\beta$-cristobalite structure, can explain the observed properties of the blue luminescence in quartz, including the very large Stokes shift, the transient volume change induced in the crystal, and the strong uniaxial fine structure. The latter we ascribe to spin-spin interaction between the two carriers when they are both present on the displaced oxygen atom, and the principal axis of the interaction is not correlated with any interatomic direction.

It is interesting to contrast the exciton self-trapping process we postulate here with the accepted picture in the alkali halides (see for example Hayes and Stoneham 1985). There it is helpful to regard the self-trapped exciton as a self-trapped hole to which an electron is bound and Itoh et al (1989b) have given arguments to suggest that the addition of the electron increases the self-trapping energy. In quartz, on the other hand, no experimental evidence has been found for hole self-trapping at temperatures 
down to $4 \mathrm{~K}$ (Hayes and Jenkin 1985, 1986); our calculations support this conclusion, as we find that on removing the electron component of the exciton the remaining hole relaxes back towards the perfect-crystal geometry. Recent observations in amorphous silica suggest that there holes may self-trap (Griscom 1989); it is possible however that effects of disorder are important in this case. The principal driving force for the exciton self-trapping in quartz appears to be the electron component; because of the admixture of silicon-oxygen antibonding states into the conduction band (Chelikowsky and Schlüter 1977), the lengthening of a silicon-oxygen bond which we postulate brings an electron state deep into the gap, while the hole, residing in a non-bonding oxygen p state, is chemically rather inert.

\section{Acknowledgments}

We are most grateful to Dr R D Amos and Dr J E Rice of the University Chemical Laboratory, Cambridge, for allowing us access to the CADPAC program, to Dr A H Harker for a number of helpful discussions and to Dr J Robertson for his comments on the manuscript. We thank Dr J P Hagon for supplying the illustration on which figure 3 is based. The computations described in this paper were carried out on the Convex-C1 of the Oxford University Computing Service. AJF was supported by a CASE studentship from the United Kingdom Science and Engineering Research Council; part of this work was supported by the Underlying Research programme of the United Kingdom Atomic Energy Authority.

\section{References}

Alonso P J, Halliburton L E, Kohnke E E and Bossoli R B 1983 J. Appl. Phys. 545396

Amos R D and Rice J E 1987 CADPAC: The Cambridge Analytic Derivatives Package Issue 4.0 (Cambridge)

Catlow C R A and Stoneham A M 1983 J. Phys. C: Solid State Phys. 164321

Chelikowsky J R and Schlüter M 1977 Phys. Rev. B 154020

Davies J J 1976 Contemp. Phys. 17275

Fisher A J 1989 DPhil Thesis University of Oxford (unpublished)

Fisher A J, Hayes W and Stoneham A M $1989 \mathrm{~J}$. Chem. Soc., Faraday Trans. II 85467

- 1990 Phys. Rev. Lett. 642667

Gillan M J 1981 Phil. Mag. A 43301

Griscom D L 1989 Phys. Rev. B 404224

Halliburton L E, Koumvakalis N, Markes M E and Martin J J 1981 J. Appl. Phys. 523565

Harker A H and Lyon S B 1979 AERE Harwell Report R-8589

Hayes W and Jenkin T J L $1985 \mathrm{~J}$. Phys. C: Solid State Phys. 18 L849

_- 1986 J. Phys. C: Solid State Phys. 196211

1988 J. Phys. C: Solid State Phys. 212391

Hayes W, Kane M J, Salminen O, Wood R L and Doherty S P 1984 J. Phys. C: Solid State Phys. 172943

Hayes W and Stoneham A M 1985 Defects and Defect Processes in Non-Metallic Solids (New York: Wiley) p 247

Hellwege K-H and Hellwege A M 1979 Elastic, Pyroelectric, Piezooptic, Electrooptic Constants and Nonlinear Dielectric Susceptibilities of Materials Numerical Data and Functional Relationships in Science and Technology, Group III, vol 11 (Berlin: Springer)

Hobbs L W and Pasucci M R 1980 J. Physique Coll. 41 C6 237

Horie H 1953 Prog. Theor. Phys. 10296

Howitt D G, Chan H W, Vance E R, De Natale J F, Hood P J and Thompson D A 1990 Radiat. Eff. Def. Solids 11239 
Itoh C, Tanimura K and Itoh N 1986 J. Phys. C: Solid State Phys. 196887 1988 J. Phys. C: Solid State Phys. 214693

Itoh C, Tanimura K, Itoh N and Itoh M 1989a Phys. Rev. B 3911183

Itoh N, Tanimura K, Stoneham A M and Harker A H 1989b J. Phys.: Condens. Matter 13911

Klein G and Chun H U 1972 Phys. Status Solidi b 49167

Lidiard A B 1981 Phil. Mag. A 43291

Pople J A and Beveridge D L 1970 Approximate Molecular Orbital Theory (New York: McGraw-Hill)

Rudra J K and Fowler W B 1987 Phys. Rev. B 358223

Shluger A L 1988 J. Phys. C: Solid State Phys. 21 L431

Shluger A L and Stefanovich E 1989 Preprint

Sigel G H 1973 J. Non-Cryst. Solids 13372

Sobel'man I I 1972 An Introduction to the Theory of Atomic Spectra (Oxford: Pergamon)

Stoneham A M 1975 Theory of Defects in Solids (Oxford: Oxford University Press) 1983 J. Phys. C: Solid State Phys. 16 L925

Tanimura K and Halliburton L E 1986 Phys. Rev.B 342933

Tanimura K, Tanaka T and Itoh N 1983 Phys. Rev. Lett. 51423

- $1984 \mathrm{Nucl}$. Instrum. Methods B 1187

Temkin D E 1970 Sov. Phys.-Solid State 111614

Tinkham M and Strandberg M W P 1954 Phys. Rev. 97937

Trukhin A N and Plaudis A E 1979 Sov. Phys.-Solid State 21644

Thiel W 1978 Theor. Chim. Acta 48357

Weeks R A 1956 J. Appl. Phys. 271376 